\title{
Thermoelectric Generation Using Industrial Grade Low-Cost Materials
}

\author{
K. P. V. B. Kobbekaduwa and N.D. Subasinghe ${ }^{*}$ \\ National Institute of Fundamental Studies, Hanthana Road, Kandy, Sri Lanka \\ ${ }^{*}$ Corresponding author
}

\begin{abstract}
Thermoelectric effect or Seebeck effect provides a means by which thermal energy can be converted into electricity directly. Voltage appears between two different types of materials (Conductor or Semiconductor) that are joined together and its junction is heated. While most efficient materials are semiconductors, these are expensive, thus, research into thermoelectric properties of industrial grade materials is very useful for fabrication of low cost devices. Using materials such as Iron, Aluminum and Brass, cheap TEGs were constructed and several parameters were tested for three separate combinations made using these materials. An output voltage up to $0.5 \mathrm{mV}$ per couple at a ZT of 0.08 was achieved. It was apparent that using active cooling methods such as ice water or heat sinks with cooling fans increased the output values to around $0.7 \mathrm{mV}$ per couple. Although the efficiencies are not high, these TEGs provide low-cost devices that can produce small-scale sustainable energy through co-generation and energy-scavenging.
\end{abstract}

Keywords- thermo electric generator; seebeck effect; thermoelectric power; low cost electricity; metals

\section{INTRODUCTION}

Finding new sources of energy is of paramount importance to fulfill the increasing demand of the world. Hence, renewable sources of energy are important as they can be naturally replenished and never run out. There are various sources of renewable energy such as sunlight, wind, rain, geothermal heat etc. and by energy conversion we can generate electricity for various applications. Thermoelectricity is one of the ways thermal energy is converted into electricity. A major advantage of thermoelectric power is that it can be generated using waste energy from various applications as well as from natural sources as long as a temperature gradient is present. The use of waste energy by the way of thermoelectric conversion include application in areas such as remote sensing, automotive, stove, geothermal, space systems and industrial power plants. Thermoelectrics can also be combined with PhotoVoltaic (PV), solar thermal or thermophotovoltaic systems [1].

A device which uses the principle of thermoelectric energy conversion is called a Thermoelectric Generator (TEG). When two dissimilar materials are joined such that two junctions are formed, an electric flow can be observed between the junctions when a temperature gradient is present between them. This phenomenon is called the thermoelectric effect or 'Seebeck effect' as it was discovered by Seebeck in 1821[2]. At the quantum level the Seebeck effect can be explained by looking at the electron-hole behavior of a material. The temperature difference between the junctions generates a change in the Fermi distribution function i.e. Fermi level. As the charge carriers near the 'hot' end are thermally excited, there will be a number of electrons which will have energies greater than the Fermi energy. These will diffuse to the 'cold' end. This means that the electric current runs towards the hightemperature end in an electron (or away from in a hole) rich material. Thus, the Seebeck current arises from the above thermal diffusion of charge carriers [3].

An important property of any TE device is its efficiency. Most of the current research work is focused on fabrication of new materials as well as understanding and improving properties of those materials to increase efficiency. Thus, most research in centered on expensive semiconductor and nano materials. This research work is more focused on developing low cost thermoelectric devices which can be manufactured using readily available industrial grade materials at an affordable cost. Most materials used are either metals or metallic alloys. Though they have relatively low Seebeck coefficients, hence lower output voltages these TEGs are still capable of producing adequate amount of power such that they can be used in a similar way to a photovoltaic cell as well as for lighting and as battery chargers.

\section{THEORY OF THERMOELECTRICITY}

\section{A. Seebeck Coefficient}

The basic theory behind thermoelectric power is described using the Seebeck coefficient,

$$
S=\frac{d V}{d T}
$$

where $\mathrm{V}$ is the voltage and $\mathrm{T}$ is the temperature.

For any TEG we use two types of materials hence we consider the relative Seebeck coefficient between them. Consider two materials A and B.

$$
V=\int_{T_{1}}^{T_{2}}\left(S_{B}(T)-S_{A}(T)\right) d T
$$

where $S_{A}$ and $S_{B}$ are Seebeck coefficients of the two materials and $\mathrm{T} 1$ and $\mathrm{T} 2$ are the temperatures of the two junctions. The Seebeck coefficients are non-linear as a function of temperature, and depend on the conductors' absolute 
temperature, material, and molecular structure. If the Seebeck coefficients are effectively constant for the measured temperature range, the above formula can be approximated as:

$$
V=\left(S_{B}-S_{A}\right)\left(T_{2}-T_{1}\right)
$$

The total voltage from a TE device is reduced further from the open circuit voltage due to internal resistance. Thus, the output voltage is given by,

$$
V_{o}=\left(S_{B}-S_{A}\right)\left(T_{2}-T_{1}\right)-I R_{T E G}
$$

where $I$ is the current and $R_{T E G}$ is the total resistance of the TE device.

\section{B. Power and Efficiency}

The total power generated can be written as,

$$
P_{T}=V_{o} I=\left(S_{B}-S_{A}\right)\left(T_{2}-T_{1}\right) I-I^{2} R_{T E G} .
$$

The efficiency of a thermoelectric device is given by,

$$
\phi=\frac{\text { Energy given to the load }}{\text { Heat energy absorbed by hot junction }}
$$

To describe the efficiency of such materials we employ a dimension less figure of merit $Z T$. Thus,

$$
Z T=\frac{\sigma S^{2} T}{\lambda} .
$$

where $\sigma$ and $\lambda$ are electrical and thermal conductivities respectively. In an actual Thermoelectric device two materials are used. Thus, the above figure is modified as,

$$
Z \bar{T}=\frac{\left(S_{B}-S_{A}\right)^{2} \bar{T}}{\left[\left(\frac{\lambda_{B}}{\sigma_{B}}\right)^{\frac{1}{2}}+\left(\frac{\lambda_{A}}{\sigma_{A}}\right)^{\frac{1}{2}}\right]^{2}} .
$$

where $\lambda_{\mathrm{A}}, \lambda_{\mathrm{B}}, \sigma_{\mathrm{A}}$ and $\sigma_{\mathrm{B}}$ are thermal and electrical conductivities of the two materials and $\bar{T}=\frac{T_{1}+T_{2}}{2}$. Using this value we can describe the maximum efficiency $\phi_{\max }$ of a TE device at max power output.

$$
\phi_{\max }=\frac{T_{2}-T_{1}}{T_{2}} \frac{\sqrt{1+Z \bar{T}}-1}{\sqrt{1+Z \bar{T}}+\frac{T_{2}}{T_{1}}}
$$

Thus, maximum efficiency is a product of the Carnot efficiency $\left(1-\frac{T_{\text {cold }}}{T_{\text {hot }}}\right)[4]$.

In this paper we discuss the potential of using some industrial grade materials to construct low cost thermoelectric devices. We test the following parameters related to the used materials.

- $\quad$ Output voltage, current and efficiency of materials

- Cooling options for suitable materials

\section{MATERIALS AND METHODOLOGY}

We used combinations of three industrial grade materials to construct junctions for thermoelectric testing. The tested junctions were,

1. Iron-Aluminum

2. Brass-Aluminum

3. Iron-Brass

All three materials were obtained as sheet metals. Galvanized iron sheets used in various everyday applications, Brass sheets used by jewelry manufacturers and Aluminum sheets used in for applications in vehicles, refrigerators etc. were the main sources. Each sheet was approximately $0.1 \mathrm{~mm}$ thick and initially $5 \times 100 \mathrm{~mm}$ metal strips were cut from the three sheets as shown in Figure 1(a). Most Iron sheets are galvanized hence it is important to remove the zinc coating before junctions are assembled.

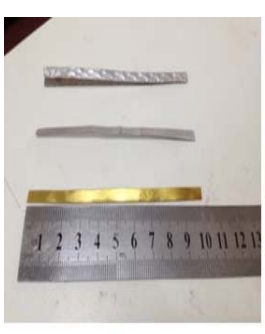

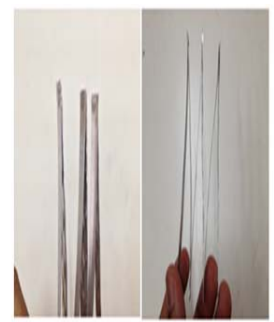

(b)

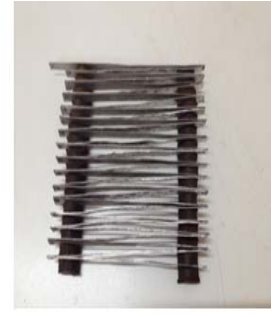

FIGURE I. (a) CUT METAL STRIPS, (b) WELDED METAL STRIPS, (c) MODULE WITH 8 PAIRS OF JUNCTIONS.

The cut metal strips were then welded together using a spot welder. For this purpose 'Telwin Digital Modular 230' spot welding machine was used. The welded strips are shown above in Figure 1(b). Eight such welded pairs of junctions make up a single module as shown in Figure 1(c). These are arranged on two clay strips which are heated such that all moisture is removed. Initially data was collected for all three types at room temperature. One end of the module was heated using a sand bath.

The experimental setup is shown in Figure 2. Temperature gradient was recorded as well as the corresponding values for voltage and resistance. Then these values were calculated for a single thermocouple. Using the data obtained cooling was only 
used on the type which had the largest output as well as the most efficient.

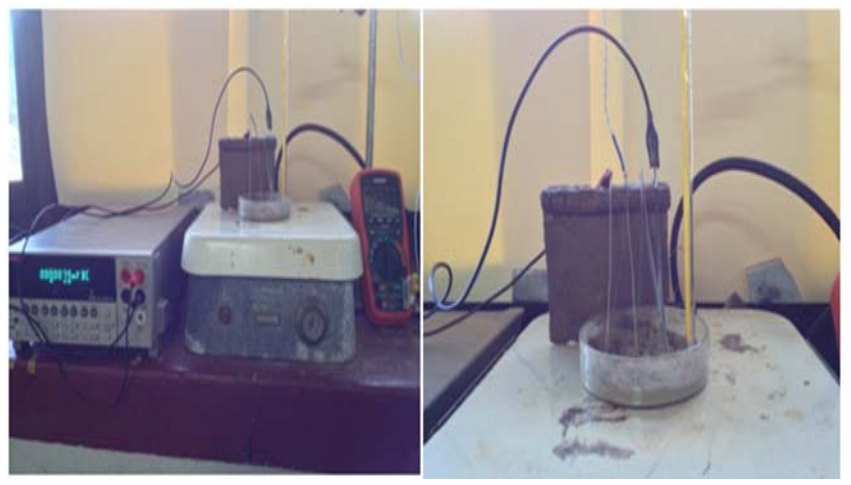

FIGURE II. EXPERIMENTAL SETUP.

The voltage, resistance and current variations obtained for all three materials are shown in Figure 3. The results show that there is a similarity in variations between Aluminum-Iron and Iron-Brass junctions. Thus, we can surmise that the properties of 'Fe' are playing a larger role in changes due to temperature in those TE junctions.

Using the above properties as well as the collected data we can calculate the figure of merit (Figure 4(A)) as well as the maximum efficiency (Figure 4(B)) associated with each material.

TABLE I. PHYSICAL PROPERTIES OF TESTED MATERIALS.

\begin{tabular}{|c|c|c|c|c|c|}
\hline Material & $\begin{array}{c}\text { Thermal } \\
\text { Expansion[4] } \\
\mathbf{x ~ 1 0} \mathbf{0}^{\mathbf{6}} / \mathbf{K}\end{array}$ & $\begin{array}{c}\text { Thermal } \\
\text { conductivity[5] } \\
\mathbf{W} / \mathbf{m K}\end{array}$ & $\begin{array}{c}\text { Specific } \\
\text { Heat } \\
\text { capacity } \\
{[\mathbf{6}] \mathbf{J} / \mathbf{k g K}}\end{array}$ & $\begin{array}{c}\text { Electrical } \\
\text { conductivity[7] } \\
\mathbf{x 1 0} \mathbf{6}^{\mathbf{\Omega m}}\end{array}$ & $\begin{array}{c}\text { Seebeck } \\
\text { coefficient[8] } \\
\boldsymbol{\mu} \mathbf{V} / \mathbf{K}\end{array}$ \\
\hline Iron & 12 & 80 & 449 & 10.3 & 19.8 \\
\hline Aluminium & 22.2 & 205 & 897 & 37.7 & 3.9 \\
\hline Brass & 18.7 & 109 & 130 & 14.1 & 6.0 \\
\hline
\end{tabular}

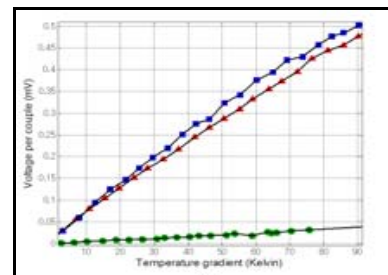

(a)

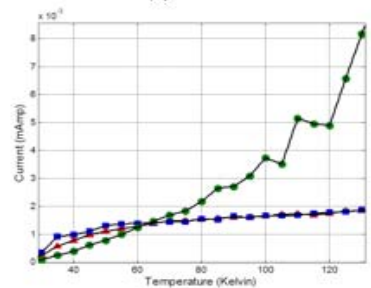

(c)

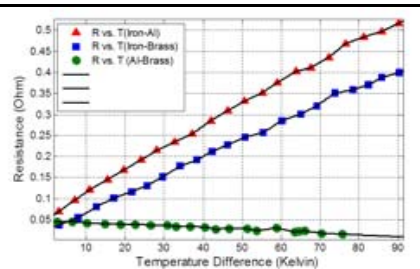

(b)

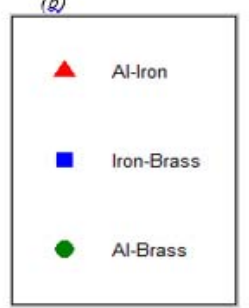

FIGURE III. (a) VOLTAGE, (b) RESISTANCE VS. TEMPERATURE GRADIENT, (c) CURRENT VS. TEMPERATURE.
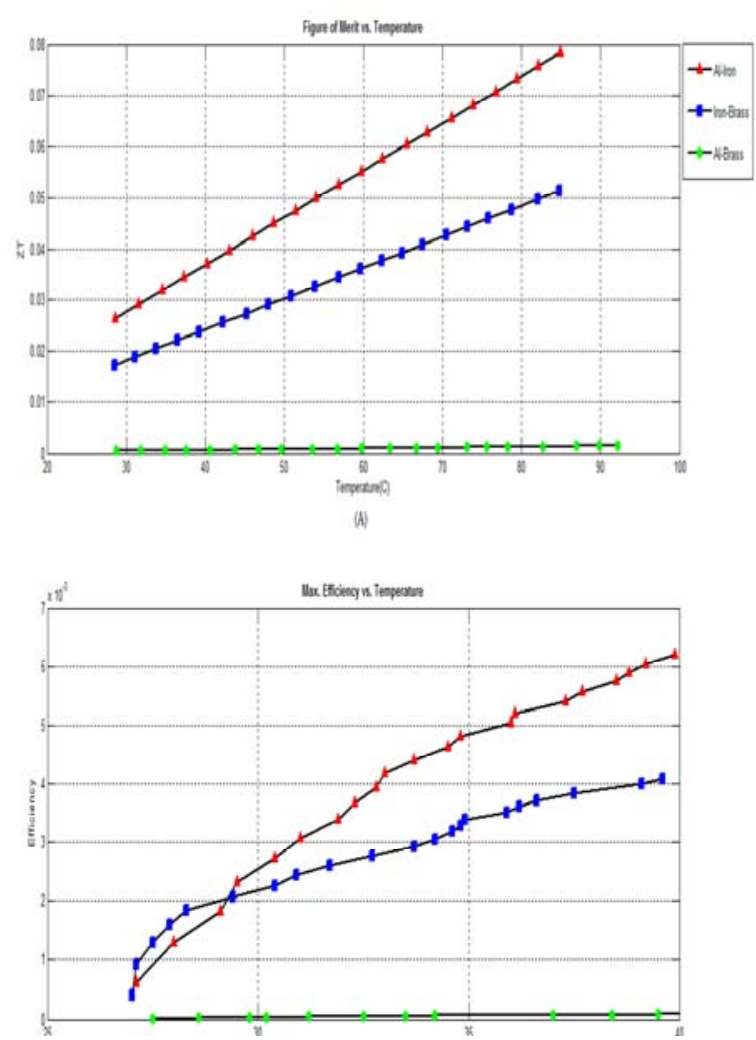

FIGURE IV. (A) FIGURE OF MERIT, (B) MAXIMUM EFFICIENCY VS. TEMPERATURE.

From these figures it appears that Iron-Brass combination produces a larger output voltage compared to the other tested junctions, but it is not the case with regard to efficiency as the Al-Iron junction is more efficient. One reason for this would be the change in internal resistance of the junctions as well as resistance variations at the welded points. Due to the close relationship between Brass - Al properties especially the Seebeck coefficients, efficiency as well as output voltages are very low, but as they have relatively large electrical conductivity values output current tend to vary unpredictably with temperature.

The most cost efficient way to obtain larger output values is to have a greater temperature gradient (From Eq. 03). Thus, we can increase the temperature at the 'hot' end or decrease it at the 'cool' end. As we cannot control the temperature at the hot end the most cost efficient way is to use cooling methods to decrease temperature. In this paper we discuss two such methods.

\section{These are,}

1. Using ice and water for cooling

2. Using a heat sink and fan to absorb access heat

There is a marked improvement in output voltage and current when using such cooling methods as shown in Figure 5. 


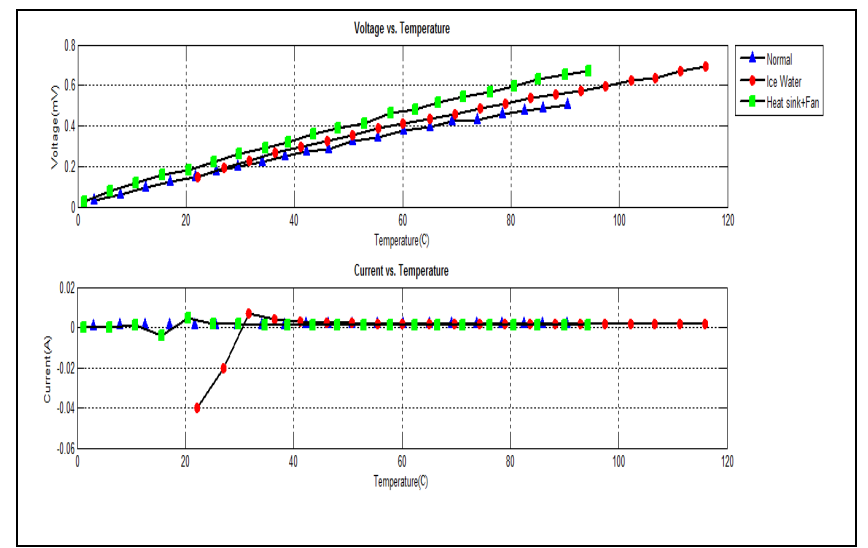

FIGURE V. (TOP) OUTPUT VOLTAGE AND (BOTTOM) CURRENT VARIATIONS WITH TEMPERATURE WITH COOLING.

These modules are able to generate a few miliamperes and milivolts worth of current and voltage respectively. With the use of active cooling we can increase those values further. There are several advantages of using these sheet metals for junction construction.

1. Cheap and readily available

2. The dimensions can be altered

3. As the sheets can directly weld together there is no thermal, resistance effects due to filler or binding materials (lead solder etc.) on the output

To increase the power output several of these modules can be connected in parallel. Several considerations should also be considered when the modules are constructed.

1. Ohmic losses due to internal resistance of the materials

\section{Thermal expansion due to high temperature}

Several solutions have been used such as clamping electronic circuits to enhance the generated voltage and consideration of thermal expansion of each individual material, in order to sustain mechanical durability [9].

\section{SUMMARY AND CONCLUSION}

To develop cheap alternatives to expensive semi-conductor thermoelectric devices industrial grade materials can be used. Materials such as Aluminum, Iron even copper and alloys such as brass, steel can be used. Using metallic sheets to construct these TE junctions gives us several advantages. As the Seebeck Coefficients are low compared to other semiconductor materials, we need several hundred junctions to generate a usable amount of power. This in turn will increase internal resistance values and cost, but as most cheap materials are metals and alloys these increases in values are manageable. Use of external circuits to enhance output power is also recommended. Using active cooling techniques can improve the output values. Heat sinks with cooling fans or ice water can be used but as the former requires external power ice is a cheaper alternative.

\section{ACKNOWLEDGMENT}

The authors gratefully acknowledge the support of the director, research as well as technical staff of the National Institute of Fundamental Studies in Sri Lanka for funding, infrastructure support as well as technical assistance in completing the current research work.

\section{REFERENCES}

[1] A. Montecucco, J. Siviter and A. R. Knox. The effect of temperature mismatch on thermoelectric generators electrically connected in series and parallel. Applied Energy, Vol. 123, p. 47-54, (2014).

[2] E. Velmre, Thomas Johann Seebeck (1770-1831). Proceedings of the Estonian Academy of Sciences and Engineering (2007), Vol. 13(4), p. 276-282

[3] S. Fujita, and A. Suzuki, Quantum Theory of Thermoelectric Power (Seebeck Coefficient). Electromotive Force and Measurement in Several Systems (2011), [online] Available at: http://www intechopen $. c 0 m /$ books/electromotive-force-andmeasurement-in-severalsystems/quantum-theory-of-thermoelectricpower-seebeck-coefficient- [Accessed 15 May 2015].

[4] Engineeringtoolbox.com, Coefficients of Linear Thermal Expansion (2015), [online] Available http://www.engineeringtoolbox.com/linear-expansion-coefficientsd_95.html [Accessed 15 May 2015].

[5] Engineeringtoolbox.com, Thermal Conductivity of some common Materials and Gases (2015), [online] Available at: $\mathrm{http}: / /$ www.engineeringtoolbox.com/thermal-conductivity-d_429.html [Accessed 15 May 2015].

[6] Engineeringtoolbox.com, Specific Heat of some common Substances (2015), [online] Available at: http://www.engineeringtoolbox.com/specific-heat-capacity-d_391.html [Accessed 15 May 2015].

[7] Engineeringtoolbox.com, Resistivity, Conductivity and Temperature Coefficients for some Common Materials (2015), [online] Available at: $\mathrm{http}: / / \mathrm{www}$.engineeringtoolbox.com/resistivity-conductivity-d 418.html [Accessed 15 May 2015]

[8] F. Cardarelli, Materials Handbook: A Concise Desktop Reference. (Springer, London, 2008), $2^{\text {nd }}$ ed.

[9] N.D. Subasinghe, N.B. Suriyaarachchi and T.B. Nimalsiri, Low-Cost Thermoelectric Power Generation Using Solar Energy, Proceedings of the International Conference on Solar Energy Materials, Solar Cells and Solar Energy Applications (Institute of Fundamental Studies, Kandy, 2011) p. 336-341. 